Pathologe 2021 · 42:147-148

https://doi.org/10.1007/s00292-021-00916-x

Angenommen: 13. Januar 2021

(c) Springer Medizin Verlag $\mathrm{GmbH}$, ein Teil von Springer Nature 2021

\author{
W. Roth ${ }^{1} \cdot$ P. Boor ${ }^{2}$ \\ 'Institut für Pathologie, Universitätsmedizin Mainz, Mainz, Deutschland \\ ${ }^{2}$ Institut für Pathologie, Universitätsklinik Aachen, Aachen, Deutschland
}

\title{
COVID-19 im Spiegel der Pathologie
}

Im September 2003 gab es schon einmal ein Editorial in Der Pathologe über Coronaviren, damals im Rahmen der relativ glimpflich verlaufenen SARS-Epidemie. Darin kam S. Ewig zur aus heutiger Sicht äußerst zutreffenden Schlussfolgerung: „Die SARS-Epidemie macht wie ihre Vorgänger auf exemplarische Weise deutlich, dass Infektionsepidemien zur permanenten latenten Bedrohung der menschlichen Gesundheit und Zivilisation gehören. [...] In der Zwischenzeit sollte die Forschung über das neue SARSCoronavirus intensiviert werden; nichts spricht dafür, dass es bei diesem Ausbruch bleiben wird“ [1]. Das damalige Editorial war überschrieben mit: „SARS ein Lehrstück der Infektionsepidemiologie und ein Meisterstück moderner Infektionskontrolle“. Die Überschrift eines aktuellen Editorials über die SARS$\mathrm{CoV}$-2-Pandemie muss angesichts des nur schwer kontrollierbaren Pandemieverlaufs wesentlich bescheidener und demütiger ausfallen.

Der ursprünglich für das vorliegende Heft geplante Titelhieß schlicht „Viruspathologie" mit dem Ziel einer allgemeinen Darstellung von pathomorphologisch relevanten Erscheinungsbildern viraler Erkrankungen. Die sich seit Anfang des Jahres 2020 überschlagenden Ereignisse der SARS-CoV-2-Pandemie führten sehr schnell dazu, dass COVID-19 der alleinige Schwerpunkt des Themenheftes wurde - mit einer überwältigenden Resonanz und Bereitschaft von Pathologen/innen, ihre Erfahrungen in Form von Artikelbeiträgen beizusteuern. Der Fachbereich Pathologie war und ist auf ganz unterschiedliche Weisen von der Pandemie betroffen. Die praktischen Auswirkungen im diagnostischen und wissenschaftlichen Be- reich wurden am Beispiel der Universitätspathologien in dieser Zeitschrift bereits thematisiert [2]. Große Verwirrung entstand in den ersten Pandemiemonaten vor allem hinsichtlich der Durchführung von Obduktionen. Auch diese Historie mit dem daraus rasch entstandenen großen öffentlichen Interesse an der Tätigkeit der Pathologie wurde bereits andernorts dargestellt und ins rechte Licht gerückt [3].

Das vorliegende Themenheft möchte der Leserschaft einerseits pathologierelevante Hintergrundinformationen $\mathrm{zu}$ COVID-19 liefern als auch praxistaugliche Hilfestellungen bieten, was die histomorphologische und molekulare Diagnostik sowie die Durchführung von COVID-19-Obduktionen betrifft. Nach einführenden Beiträgen über die Biologie und Pathologie von Coronaviren (K. Klingel et al.) und die klinischen sowie systemischen Krankheitseffekte (Lax et al.) werden die Auswirkungen einer COVID-19-Erkrankung auf die wesentlichen Organsysteme von Lunge und Herz (Ackermann et al.), ZNS (Acker et al.), Nieren (Amann et al.) und lymphatisches System (Tzankov et al.) beleuchtet. In weiteren zusammenfassenden Artikeln werden die praktischen Aspekte der Obduktionsdurchführung (Märkl et al.) sowie die gewebebasierten Nachweismethoden von SARS-CoV-2 (Boor et al.) behandelt. In diesem Zusammenhang ist die Etablierung des Deutschen Registers für COVID-19-Obduktionen (DeRegCOVID) und des Deutschen Forschungsnetzwerks Autopsien bei Pandemien (DEFEAT PANDEMIcs) im Rahmen des Nationalen Forschungsnetzwerkes der Universitätsmedizin zu COVID-19 von besonderer Bedeutung, 
um strukturierte Daten zu COVID-19Obduktionen zu erfassen, harmonisierte Leitlinien zur Durchführung von $\mathrm{Au}-$ topsien $\mathrm{zu}$ entwickeln und somit die Ausgangsbedingungen innerhalb der Pathologie für zukünftige Epidemien und Pandemien im Sinne einer Krisenprävention $\mathrm{zu}$ verbessern (von Stillfried et al.). Schließlich wendet sich eine Arbeit noch der Fragestellung zu, welche Auswirkungen die COVID-19-Pandemie auf das weltweite wissenschaftliche Publikationswesen hat und welche Rolle hierbei das Fachgebiet der Pathologie spielt (Strobl et al.).

Uns allen bleibt zu hoffen, dass der Titel eines zukünftigen Artikels nach hoffentlich erfolgreich überstandener COVID-19-Pandemie ähnlich optimistisch formuliert werden kann wie die oben zitierte Überschrift von S. Ewig. Für die Pathologie ergibt sich in der Zwischenzeit die Herausforderung, durch ein verbessertes Verständnis der Pathogenese und durch innovative Diagnostik einen wesentlichen Teil dazu beizutragen.

Prof. Dr. W. Roth

Prof. Dr. P. Boor

\section{Korrespondenzadresse}

\section{Prof. Dr. W. Roth}

Institut für Pathologie, Universitätsmedizin

Mainz

Langenbeckstr. 1, 55131 Mainz, Deutschland

wilfried.roth@unimedizin-mainz.de

Prof. Dr. P. Boor

Institut für Pathologie, Universitätsklinik

Aachen

Pauwelsstraße 30, 52074 Aachen, Deutschland

pboor@ukaachen.de

Interessenkonflikt. W. Roth und P. Boor geben an, dass kein Interessenkonflikt besteht.

\section{Literatur}

1. Ewig S (2003) SARS - ein Lehrstück der Infektionsepidemiologie und ein Meisterstück moderner Infektionskontrolle. Pathologe 24:335-337. https:// doi.org/10.1007/s00292-003-0637-6

2. Schreck J, Baretton G, Schirmacher P (2020) Situation der Deutschen Universitätspathologien unter den Einschränkungen der Corona-Pandemie - Auswertung einer ersten repräsentativen Umfrage. Pathologe 41:400-405. https://doi.org/ 10.1007/s00292-020-00791-y

3. Bundesverband Deutscher Pathologen (2020) Mitteilungen des Bundesverbandes Deutscher
Pathologen. Pathologe 41:425-427. https://doi. org/10.1007/s00292-020-00798-5
Ausschreibung:HochgesandPreis für Pathologie

Die öffentliche, gemeinnützige Hochgesand-Stiftung für Pathologie schreibt 2021 wieder einen Preis in Höhe von $10.000 €$ zur Förderung der Forschung im Bereich der Pathologie aus.

Hintergrund: Der Geheime Medizinalrat Dr. Jakob Hochgesand ermöglichte durch eine großzügige Stiftung an die Stadt Mainz die Gründung des ersten Institutes für Pathologie im Jahre 1914. In dieser Tradition wurde 2016 die zweite Hochgesand-Stiftung durch die Nachfahren des Jakob Hochgesand, der Familie Hochgesand, begründet. Sie soll die Erinnerung an den großen Förderer der Pathologie Jakob Hochgesand wachhalten. Weitere Informationen zur Stiftung finden sich auf der Website www.hochgesandstiftung.de.

Teilnahmebedingungen: Durch den Preis soll ein/e junge/r, wissenschaftlich aktive/r Pathologin bzw. Pathologe (in Weiterbildung oder Facharzt) für eine zwischen 2019 und 2021 publizierte oder zur Publikation angenommene herausragende Arbeit ausgezeichnet werden. Die wissenschaftliche Arbeit sollte überwiegend in einem Institut für Pathologie im deutschsprachigen Raum durchgeführt und nicht bereits zuvor mit einem anderen Preis ausgezeichnet worden sein. Das Thema der Arbeit kann grundlagenwissenschaftlich oder diagnostisch/therapeutisch anwendungsbezogen sein. Der/die Bewerber/in sollte in der Regel zum Zeitpunkt der Bewerbung nicht älter als 35 Jahre sein (Ausnahmen erfordern eine Begründung). Eigenbewerbungen sind möglich und erwünscht. Neben der Publikation soll ein kurzes, die Arbeit zusammenfassendes Anschreiben (1-2 Seiten), ein Lebenslauf und eine Publikationsliste eingereicht werden.

Alle Unterlagen sollen elektronisch eingereicht werden beim Schriftführer des Kuratoriums der Hochgesand-Stiftung, Herr Prof. Dr. Wilfried Roth, Direktor des Institutes für Pathologie der Universitätsmedizin Mainz, Langenbeckstr. 1, 55131 Mainz, E-Mail: Wilfried.Roth@unimedizinmainz.de. Der Einsendeschluss ist der 30.06.2021. 